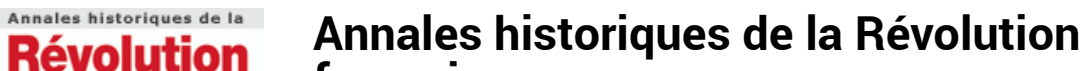

française française

317 | juillet-septembre 1999

Numéro spécial : France-Allemagne. Interactions,

références

\section{Réflexions anglaises sur la Révolution de France}

\section{Pascal Dupuy}

\section{(2) OpenEdition}

10 Journals

\section{Édition électronique}

URL : https://journals.openedition.org/ahrf/1985

DOI : 10.4000/ahrf.1985

ISSN : 1952-403X

Éditeur :

Armand Colin, Société des études robespierristes

\section{Édition imprimée}

Date de publication : 1 septembre 1999

Pagination : 537-542

ISSN : 0003-4436

\section{Référence électronique}

Pascal Dupuy, «Réflexions anglaises sur la Révolution de France », Annales historiques de la Révolution française [En ligne], 317 | juillet-septembre 1999, mis en ligne le 11 avril 2006, consulté le 23 avril 2022. URL : http://journals.openedition.org/ahrf/1985; DOI : https://doi.org/10.4000/ahrf.1985

Ce document a été généré automatiquement le 23 avril 2022.

Tous droits réservés 


\title{
Réflexions anglaises sur la Révolution de France ${ }^{1}$
}

\author{
Pascal Dupuy
}

Proposée et dirigée par le Professeur Claude Mazauric, cette étude de la représentation de la France et des Français à travers la caricature anglaise à la fin du XvIII ${ }^{\mathrm{e}}$ siècle, doit aussi à Colin Lucas, de l'Université d'Oxford, une dette intellectuelle. Dans un petit café proche du British Museum où nous nous étions donné rendez-vous, il écouta mon projet et, approuvant mes remarques à propos des apories de l'historiographie anglaise sur le sujet, il m'encouragea à concentrer ma recherche sur les gravures satiriques anglaises relatives à la Révolution française. Il modéra cependant mon enthousiasme de jeune chercheur avide de sources, d'histoire globale et d'enquêtes croisées me suggérant de porter plutôt mon attention sur la vie et l'œuvre d'un seul caricaturiste, James Gillray. Au terme de ce travail, j'ai le sentiment d'être retombé, infidèlement à l'égard de Colin Lucas, dans mes vieux démons et d'avoir utilisé toutes les sources que j'ai pu rencontrer au cours de mes périples afin d'apprécier l'écho de la Révolution française en Grande-Bretagne mais aussi d'éclairer, d'animer et surtout de comprendre des images aux références multiples, aux sens confus, aux significations immédiates souvent oubliées ou aux accents perdus par le temps. Pouvait-il cependant en être autrement? En d'autres termes, la source iconographique utilisée comme matière première d'une démarche historique peut-elle faire l'économie de la confrontation avec les sources discursives, de la mise en parallèle, de l'effet de miroir ou du regard croisé ? Nous ne le croyons pas et toute notre recherche fut entreprise à partir de cet a priori de méthode. Toutefois dans le même temps, cet axe déterminant de notre approche méthodologique se trouvait inséré dans un projet encore plus vaste qui consistait, en autonomisant la source iconographique, à repérer, inventorier et cataloguer la totalité des caricatures anglaises relatives à la France en révolution et à la France révolutionnaire et publiées en Grande-Bretagne entre 1789 et 1802. Cette démarche nous l'avons voulue systématique, ce qui nous a obligé à de multiples déplacements en Europe et également aux États-Unis où se trouvent de nombreuses et importantes collections de caricatures anglaises, comme le notent souvent avec regret les conservateurs britanniques. L'une des difficultés principales que nous avons 
rencontrées au cours de cette enquête des sources réside dans l'éparpillement des collections et dans l'insuffisance des catalogues indexés en renvoi à celui du British Museum, ce qui nous a très souvent obligé à étudier des collections entières pour des résultats négligeables ou insignifiants.

2 Pour l'index exhaustif à triple entrée de la Lewis Walpole Library de l'Université de Yale, combien de collections non microfilmées, non inventoriées et empilées en vrac dans des cartons? Malgré ces difficultés, sur lesquelles nous reviendrons, nous avons tout de même pu relever 838 caricatures relatives à la Révolution française dont nous pouvons pratiquement assurer qu'elles furent toutes publiées entre 1789 et 1802. Sur cette masse de gravures, reproduites dans sa quasi-totalité dans notre Tome II et cataloguées chronologiquement par collection (British Museum- Collections Internationales), nous avons exhumé 245 caricatures peu utilisées voire inconnues et qui jusqu'à maintenant n'avaient qu'extrêmement rarement fait l'objet d'une attention particulière, d'une analyse critique ou d'un quelconque commentaire. Pour ce qui est de l'année 1792 par exemple, année phare et décisive en France puisque c'est alors que disparaît la royauté multiséculaire, mais également moment de tension politique exacerbée en Grande-Bretagne, les gravures que nous avons repérées dans plus d'une dizaine de collections différentes, atteignent pratiquement le nombre d'estampes connues et consultables à ce jour au British Museum. Ces 245 gravures "nouvelles " refondues dans le fond du British Museum, sans modifier fondamentalement, sauf patrimonialement, ce que l'on savait ou ce que l'on croyait savoir de la réaction graphique satirique anglaise à la Révolution française, permettent toutefois d'apporter des nuances et des inflexions dans l'analyse. Il est ainsi troublant de constater qu'un bon nombre de ces gravures jusqu'alors inexploitées marquent des sentiments radicaux, voire profrançais, insoupçonnés et insoupçonnables, en particulier jusqu'en 1793. Au contraire, plus on progresse dans la chronologie de la Révolution, plus ces gravures contestataires se font discrètes et plus les caricatures loyalistes et progouvernementales envahissent l'espace public, en réaction prophylactique justement à une possible invasion française honnie et redoutée.

3 Cette recherche méthodique, malgré sa lenteur et ses résultats parfois décevants, nous a familiarisé, par une confrontation régulière, avec l'ensemble de la production graphique satirique anglaise et nous a permis d'appréhender l'alphabet symbolique, la codification allégorique, les effets péjoratifs et mélioratifs des signaux venus du temps long. Si la rupture essentielle de l'événement révolutionnaire est certes demeurée notre point d'ancrage privilégié, nous avons cependant élargi chronologiquement, quantitativement et qualitativement, l'objet étudié afin de comprendre les éléments qui demeuraient nets, flous, ou qui disparaissaient de l'objectif pour reprendre un vocabulaire photographique. Cet instantané de la séquence révolutionnaire et de ses appendices que nous avons analysé se devait d'être replacé dans un plan plus large: celui de la lente fabrication d'un inventaire des signes qui, depuis William Hogarth, hante l'univers grotesque et satirique de la caricature politique anglaise. Sans cette immersion référentielle et symbologique, l'événement révolutionnaire français aurait perdu de sa force, de son pouvoir et de son impact ou au contraire serait devenu un objet monstrueux, difforme et effrayant. C'est aussi pour cette raison que nous avons décidé, sous l'impulsion de notre directeur de recherches, d'inventorier sous la forme d'un catalogue informatisé chaque image repérée, à partir d'un faisceau de questions afin de rendre au plus près son contenu technique, idéologique, politique et 
symbolique. Cet apport informatique devait à la fois nous donner des indications précises et exactes des flux et des reflux, des remous, des vagues et des tempêtes qui soufflent sur l'ensemble de la production de caricatures anglaises relatives à la Révolution française, mais également corriger les inflexions ou les aberrations inhérentes à une simple méthode qualitative. Travail fastidieux, mais qui nous a donné un accès privilégié, en profondeur, à l'intimité de chaque gravure, à ses signes, ses forces et ses faiblesses, à ses dits et ses non-dits, et, au risque de paraitre pompeux, à ses « sentiments". Chacune des 838 caricatures qui composent notre corpus, possède une émotion que nous n'avons cependant que très rarement interprétée à partir d'un discours théorique esthétique. Notre seule incursion dans ce domaine fut de préciser les étapes de la naissance de la caricature afin d'expliquer son épanouissement en Grande-Bretagne à la fin du XVIII siècle. Ici encore, la prise en compte du temps plus long nous a permis d'appréhender les ressorts et le fonctionnement général du mécanisme de la caricature. Dans la mesure où, comme s'accordent à le penser tous les spécialistes, la production anglaise des années 1780-1800 représente probablement la forme la plus aboutie et articulée de la technique caricaturale, il nous a semblé indispensable, afin de mesurer ses réactions, de saisir la complexité de ses rouages. La compréhension historique et esthétique de la caricature a renforcé nos hypothèses relatives aux pouvoirs de l'image. Cette notion des effets de l'image, et en ce qui nous concerne, de la caricature, a accaparé une partie de notre recherche et dirigé partiellement notre approche méthodologique. Nous avons essayé, comme le proposait Louis Marin en 1993 dans un ouvrage posthume (Des pouvoirs de l'image. Gloses), tout en soulignant le caractère quelque peu utopique de ce principe, de connaitre la force de l'image à partir d'une reconnaissance de ses effets, " en les lisant dans les signaux de leur exercice sur les corps regardants et en les interprétant dans les textes où ces signaux sont écrits dans les discours qui les enregistrent, les racontent, les transmettent et les amplifient jusqu'à capter quelque chose de la force qui les a produits ». Dans cette optique, les récits de voyage nous sont apparus comme des intermédiaires précieux favorisant l'évocation de la chose vue comme du sentiment personnel. Le caractère ambigu de ces textes, où l'auteur décrit et raconte pour mieux se raconter et se mettre en scène, nous a paru une source idéale pour donner simultanément des détails pratiques sur la production et la diffusion des caricatures, mais également pour dévoiler ses effets sur l'inconscient collectif. Les pamphlets et la presse, les autres sources ici privilégiées, se révèlent être d'un usage plus traditionnel, dans la mesure où elles accompagnent ou sont accompagnées d'images, et en raison de leur utilisation par d'autres historiens " iconophiles " avant nous, comme Michel Vovelle, Claude Langlois ou encore Annie Duprat. Elles ont, en effet, éclairé un peu plus les caricatures en les dévoilant parfois, en les rendant plus accessibles et compréhensibles toujours. Ces sources croisées, parallèles, complémentaires, dessinant des entrelacs et puisées en France comme en Grande-Bretagne, ont réfléchi la portée des caricatures et nous ont permis d'atteindre les effets et les indices de la puissance de l'image. Elles nous ont également préservés, du moins le croyons-nous, des dangers, soulignés récemment par Carlo Ginzburg (Le Juge et l'Historien, 1998 pour la traduction française), d'une histoire des représentations « du vide » trop éloignée d'une réalité historique, qui serait certes impossible à saisir, mais que l'historien se doit légitimement, cependant, d'essayer de prendre en charge, voire d'atteindre à travers les procédés de la connaissance indirecte. Grâce à l'apport de ces différentes sources étudiées en symbiose avec les images, il nous est apparu que la production caricaturale 
anglaise relative à la France en révolution, participait de tout son poids graphique et idéologique à l'élaboration d'un discours, à l'alimentation d'une opinion publique avide d'informations, à la construction identitaire d'une nation en formation et alors en forte expansion, ainsi qu'à la construction d'une image déformante à partir d'une mise en scène théatrale et imaginaire des événements français où le drame succède à la comédie.

Outre notre corpus, uniquement composé de gravures publiées en Grande-Bretagne entre 1789 et 1802, nous avons aussi repéré, pour la France et les États-Unis systématiquement, pour d'autres pays par effets de coups de sonde, les caricatures anglaises adaptées, traduites ou ayant fait l'objet d'une copie partielle à l'étranger. Cette centaine de caricatures françaises, italiennes, allemandes, suisses, hollandaises, russes ou américaines pour la plupart reproduites sous la forme d'illustrations à la fin des trois volumes qui composent notre Tome I, indiquent sans détour la force expressive de la production britannique, précisent le degré d'admiration qu'elle suscitait en Europe et dans le monde à la fin du XviII ${ }^{\mathrm{e}}$ siècle, sa vitalité esthétique et la profondeur anglaise et protestante de son propos, même s'il peut arriver que son message originel soit quelquefois détourné.

Malgré notre incapacité à donner des évaluations précises de la production totale de caricatures publiées en Grande-Bretagne entre 1789 et 1802, pour des raisons d'éparpillement et de négligences institutionnelles évoquées plus haut, nous pouvons cependant avancer, avec une marge minimale d'erreur, que les 838 caricatures formant notre corpus représentent près de la moitié des gravures politiques publiées pendant la période étudiée. Cette dimension quantitative renforce nos hypothèses préalables d'un discours idéologique à portée politique de la caricature. Dans le contexte de la production et de la diffusion des estampes, et malgré la relative liberté dont jouissait le graveur britannique, ces données brutes indiquent également les évidentes relations que la caricature était contrainte d'entretenir avec le pouvoir. Pouvoir monarchique, pouvoir de l'État, par l'intermédiaire de ses plus hauts représentants, mais également pouvoir des partis politiques, représentant eux-mêmes le pouvoir de l'argent et de la propriété. La caricature, et tout particulièrement ses créateurs les plus talentueux, a fini par s'engager avec force, si ce n'est avec conviction, dans le combat contrerévolutionnaire. Elle fut, comme le montrent les archives des associations loyalistes, un objet d'attention et de tension qui se devait d'être dirigé, surveillé, contrôlé afin d'en garantir les effets potentiels. Commandes ouvertes ou détournées, censure délibérée ou indirecte, s'abattent sur l'estampe satirique qui devient un objet obscur du désir tout en véhiculant dans ses grandes lignes un message de force et de répulsion. Dans sa dimension productive générale comme dans ses données particulières, humaines et chronologiques, la caricature anglaise rappelle son intérêt pour les événements de France. Ainsi l'artiste le plus prolifique de notre catalogue reste James Gillray qui fut pendant quelques années au service du gouvernement et engagé dans diverses associations loyalistes. De plus, derrière cette figure de proue, gourou véritable de la profession à la fin du XviII ${ }^{e}$ siècle par la force de son talent et de sa verve, les autres caricaturistes ont souvent des relations privilégiées avec les dirigeants du pays et leurs satellites ou avec les représentants de l'opposition whig et radicale. Enfin, les imprimeurs-libraires, dont la fonction est aussi marquée par la médiation commerciale, soulignent ces traits et favorisent, qui une production contre-révolutionnaire, qui une production radicale. De même, 1798 et 1793 représentent les pics de production les plus importants et s'expliquent par les objectifs antagonistes qui se cristallisent pendant ces 
deux années entre les différents protagonistes du débat politique britannique et dans une moindre mesure français. Cette respiration décousue, ces effets fluctuants, on les retrouve avec une grande netteté et symétrie dans les autres sources étudiées, en particulier dans la production pamphlétaire qui faisait l'objet, comme les caricatures, d'une attention et d'une bataille d'opinion considérables. La presse quotidienne renvoie également à des rythmes saccadés semblables et participe de front à la lente construction d'un discours mobilisateur antifrançais. Ces trois productions, caricatures, pamphlets et presse, auxquelles on peut associer d'autres outils de communication, tels que les sermons ou les romans historiques, porteurs, eux aussi, d'une identité intellectuelle et d'un message idéologique, participent, en un processus dynamique, à une forme d'acculturation politique qui progressivement envahit le monde social et culturel britannique. Au même titre que les journaux, la caricature est source d'information et permet à ceux qui en ont connaissance de savoir, de réfléchir et de penser. Comme le notait Roger Chartier en 1989 [in (dir. par) Michel Vovelle, L'image de la Révolution française, 1990] pour le cas français « pendant la Révolution, l'événement est ce que les récits en disent, et la légitimation ou la dépréciation des uns et des autres dépend directement des images les plus puissantes et les mieux reçues. Pour les partis en lutte, il s'agit donc avant tout d'imposer comme vrai ce que chacun entend donner à croire, d'établir comme hégémonique sa perception de la lutte politique comme de l'affrontement social ». Cette réalité des représentations, si elle est en partie altérée en France par un engagement concret et quotidien susceptible de modifier un jugement artificiel imposé, est en Grande-Bretagne d'une présence et d'une prégnance incontournables. L'effet de distance, puis la guerre, à partir de 1793, entre les deux nations, ainsi qu'une volonté délibérée de parodie, renforcent les effets troubles et de distorsion-déformation, inhérents à la technique de la caricature, jusqu'à les rendre invisibles au public. Entouré d'objets et d'informations détournés, le lecteur-spectateur finit par accepter les représentations parodiques qui lui sont proposées, d'autant qu'elles lui sont le plus souvent familières puisqu'elles sont issues d'un argumentaire visuel ancien et traditionnel. La place prépondérante directe ou par rebond de la Révolution française dans les différentes formes du débat politique en Grande-Bretagne constitue une volonté de discrédit ou, au contraire, de légitimité d'un événement porteur de modernité. Mais plus encore, la Révolution est surtout ressentie ou utilisée comme un élément de comparaison qui doit renforcer l'idée du bonheur d'être britannique et plus singulièrement d'être né anglais. Cette notion, déjà présente sous l'Ancien Régime dans le contenu des caricatures anglaises relatives à la France, traverse nos 838 gravures. Pour la plupart de ces œuvres, et donc pour leur(s) auteur(s) et surtout pour leur public, l'événement français n'est qu'un prétexte à développer des sentiments pro ou antifrançais, eux-mêmes porteurs d'un message identitaire de type " nationaliste ». La comparaison, autre élément fondamental de la technique de la caricature, entre les deux nations est alors utilisée à répétition afin de souligner les errements politiques de l'une et la direction raisonnée de l'autre, tout en rendant simultanément la philosophie rationaliste des Lumières responsable du déclenchement de la Révolution. C'est ce que Gerald Newman appelle le « stéréotype Gaulois » et qui sera l'un des moteurs du nationalisme anglais au début du XIX ${ }^{e}$ siècle. Ces effets de contraste vont surtout s'appuyer sur certains éléments clés de la vie politique, sociale, économique et culturelle de la Grande-Bretagne et contribuer à délaisser, à quelques exceptions près, la représentation du déroulement des événements révolutionnaires français. Si la prise de la Bastille, la fuite à Varennes, la mort du roi et les affrontements 
armés sont logiquement commentés, ce sont surtout les débats idéologiques et politiques britanniques qui sont portés à l'écran de la caricature. La représentation de la France et des Français joue un rôle de révélateur dans le développement de l'image idéale présentée par les gravures. Bien sûr, l'auto-satire n'est pas absente de cette imagerie, à travers par exemple les personnages de John Bull et de George III d'ailleurs souvent associés voire mêlés, mais elle sert, ici encore, à glorifier un régime capable d'accepter la dérision, la raillerie et l'humour, garants des libertés anglaises et du débat politique britannique. La Révolution évacuée, ou tout au moins résumée à quelques journées ou événements paroxystiques, il restait à utiliser par les artistes-graveurs, pour leur public, ce qui constituait l'ensemble des valeurs fondamentales de la société anglaise. La religion dans cet inventaire symbolique et politique prend la part du lion, cet animal symbolisant par ailleurs, quant à lui, dans les caricatures, comme dans l'iconographie emblématique, la puissance de la monarchie britannique face au coq ou, un peu plus tard, aux grenouilles françaises. De même, la foule urbaine, policée et active en Angleterre, s'oppose à l'anarchie sanguinaire du peuple français, oisif et dangereux. Paris efface les pompes monarchiques de Versailles et devient le décor unique et populaire de la société révolutionnaire. Elle abrite la foule des sans-culottes ainsi que la guillotine, symbole éloquent du message réducteur de l'idéologie révolutionnaire française. Les signes fantasmés ou issus du mouvement révolutionnaire français jouent également un rôle dans l'espace symbolique de la caricature, leur multiplication évocatrice renforçant le caractère inéluctable de leur fonction, tout en connotant péjorativement les personnages auxquels ils sont associés. Toujours dans ce champ des signes, la peur de l'invasion sert également d'épouvantail idéologique et condamne l'ennemi intérieur, tous ceux qui en Grande-Bretagne pourraient par leurs agissements politiques favoriser un débarquement. Ici encore, on touche à une notion dépassant largement le temps court de la Révolution et particulièrement sensible en Angleterre, avant et bien après la parenthèse révolutionnaire française.

6 On peut ainsi, à titre d'exemple, s'intéresser à un auteur incontournable de la littérature et de la culture anglaise, William Shakespeare, dont l'œuvre est largement présente sous forme de citations, d'évocations ou de parodies dans la production caricaturale de la deuxième moitié du XvIII ${ }^{e}$ siècle. La Tempête, sa dernière pièce, en forme de testament poétique, nous permet de mettre en évidence certains thèmes développés par les caricatures comme faisant partie intégrante de l'inconscient collectif et de l'imaginaire culturel anglais. Ainsi la tempête elle-même qui ouvre la pièce, symbole violent d'une rupture où l'ordre ne règne plus, peut être comparée, presque deux siècles plus tard, aux effets traumatiques d'une révolution. Thomas Carlyle au XIX ${ }^{e}$ siècle, comme Joseph de Maistre, utiliseront d'ailleurs souvent cette analogie de la " grande tourmente " pour décrire le mouvement révolutionnaire français. À l'opposé, l'île magique, métaphore de l'insularité britannique, qui abrite le roi, devient le point d'ancrage de la société en tenant tête aux forces naturelles. Enfin, les personnages mêmes, et tout particulièrement ceux censés représenter la malveillance, peuvent être réutilisés dans une mise en scène opposant la France révolutionnaire à la Grande-Bretagne monarchique. Ainsi Caliban, à la nature brutale, renvoie aux sans-culottes français, tandis qu'Antonio qui se sert de son éducation et de son rang pour son action malfaisante, peut être associé à ceux qui, comme Charles James Fox en Grande-Bretagne, sont censés fomenter une révolution intérieure. Plusieurs gravures de notre catalogue utilisent cette trame allégorique en une couche 
supplémentaire de sédimentation symbolique qu'il nous a fallu distinguer et apprendre à reconnaître sous peine de perdre un niveau de lecture.

7 Toutefois, alors que se composait progressivement un répertoire de signes augmenté par le temps et les événements, le temps court de la Révolution accélérait une nouvelle association satirique de formes et de codes relatifs à la France et aux Français, qui allaient bientôt envahir l'univers social et politique anglo-saxon. Issus des caricatures anglaises publiées à la fin du XVIII ${ }^{e}$ siècle en réaction aux événements révolutionnaires français, ces stéréotypes vont se propager à travers la littérature « industrielle » du XIX siècle, puis au $\mathrm{xx}^{\mathrm{e}}$ siècle par l'intermédiaire de la fiction cinématographique ou des romans populaires, comme ceux de la Baronne d'Orczy et de son héros récurrent, le Mouron rouge. Tous ces auteurs, de George Cruikshank à Charles Dickens en passant par Thomas Carlyle et les cinéastes anglo-saxons du xxe siècle, ont ceci de commun avec les caricaturistes anglais de l'époque révolutionnaire, qu'ils luttent contre des mouvements de protestation et de contestation émaillant, avec plus ou moins d'intensité, l'histoire des deux siècles qui nous séparent de la Révolution française. Leur volonté commune de recourir à cet important arsenal symbolique et satirique contrerévolutionnaire révèle la richesse et la force expressive d'un fond jusqu'alors exploité mais non systématiquement exploré, et marque aussi la volonté, au travers d'un discours fondé sur la diffamation et le mode péjoratif, de proposer un contre-modèle conservateur au discours émancipateur de la Révolution française.

\section{NOTES}

1.Notre thèse, «L'Angleterre face à la Révolution: la Représentation de la France et des Français à travers la caricature, (1789-1802) », fut soutenue le 11 décembre 1998 à l'Université de Rouen devant MM les Professeurs Jean-Marie Constant (Université du Maine), Claude Langlois (École Pratique des Hautes Études), John Merriman (Yale University), Claude Mazauric (Directeur de la recherche, Université de Rouen), Michel Vovelle (Université de Paris I Panthéon Sorbonne). Mention très honorable avec félicitations à l'unanimité.

\section{AUTEUR}

PASCAL DUPUY

Université de Rouen - I.R.E.D.-C.R.H.C.T. 